\title{
Reabilitação após lesão do nervo radial por fratura cominutiva de úmero: um estudo de caso
}

\author{
Ariane Bôlla Freire* Amanda Albiero Real* Nathália Mezadri \\ Pozzebon* Marina Segala* Jefferson Potiguara de Moraes ${ }^{* *}$
}

Resumo: Introdução - As fraturas cominutivas da diáfise umeral são muito freqüentes em acidentes. Como principais complicações, citam-se as lesões do nervo radial. Objetivo - descrever os resultados da intervenção fisioterapêutica com a Estimulação Elétrica Funcional (FES), estímulos proprioceptivos e cinesioterapia progressiva em uma paciente com lesão do nervo radial, após fratura cominutiva de úmero. Metodologia - estudo de caso com análise descritiva. A avaliação foi composta por uma ficha de anamnese e exame físico. 0 tratamento, composto por 2 sessões semanais, com duração de 1 hora cada, totalizando 16 sessões, incluía estimulação da inervação, exercícios progressivos de fortalecimento muscular e funcionais. Resultados - paciente apresentou aumento de força muscular, da Amplitude de Movimento (ADM), principalmente de punho e articulação Metacarpofalangeana (MCF). $O$ uso da FES mostrou-se eficaz na estimulação da musculatura extensora do punho. Conclusão - a utilização da eletroestimulação e cinesioterapia progressiva apresentaram resultados positivos no tratamento dessa paciente, restaurando a sua ADM e força muscular.

Descritores: Fraturas Cominutivas; Nervo Radial; Reabilitação; Eletroterapia; Técnicas Fisioterápicas.

\section{Rehabilitation of injury after radial nerve comminuted fracture of humerus: \\ a case study}

Abstract: Introduction - Comminuted fractures of the humeral diaphysis are very frequent in accidents. The major complications are the radial nerve lesions. Objective - to describe the results of physical therapy intervention with FES, proprioceptive stimulus and progressive kinesiotherapy in a patient with radial nerve injury after comminuted fracture of humerus. Methodology - a case study with descriptive analysis. The physical therapy evaluation was composed of a sheet of anamnesis and physical examination. The treatment was consisted of 2 sessions per week, with the duration of 1 hour each, totaling 16 sessions, and included stimulation of nerve fibers (FES), as well as progressive muscle strengthening exercises and functional. Results - patient showed gain of muscle strength, increased ADM, especially wrist and metacarpophalangeal joint. The use of FES was effective in stimulating the extensor muscles of the wrist. Conclusion - the use of electrical stimulation and progressive kinesiotherapy showed positive results in treating this patient, restoring her range of motion and muscle strength. Descriptors: comminuted fracture; injuries; radial nerve; rehabilitation, functional electrical stimulation, physical therapy techniques.

Descriptors: Comminuted Fracture; Radial Nerve; Rehabilitation, Electrotherapy, Physical Therapy Techniques.

\footnotetext{
* Graduanda em Fisioterapia na Universidade Federal de Santa Maria (UFSM), Santa Maria, RS, Brasil.

**Mestre em Ciências do Movimento Humano pela Universidade do Estado de Santa Catarina (UDESC), Florianópolis, RS, Brasil.
} 


\section{Introdução}

Os politraumas são em sua grande maioria causados por acidentes de trânsito e caracterizam-se por uma síndrome decorrente de lesões múltiplas, com reações sistêmicas seqüenciais que podem levar à falha ou a disfunção de órgãos ou sistemas vitais não diretamente lesados ${ }^{1}$. As regiões corpóreas mais freqüentemente atingidas são os membros inferiores e superiores ${ }^{2}$. Dentre as fraturas nos membros superiores, as cominutivas da diáfise umeral são as mais comuns nos acidentes de alto impacto. Nesses casos o tratamento cirúrgico é o mais indicado, podendo ser fixadas, a critério dos cirurgiões, através de placas e parafusos ou de hastes intramedulares ${ }^{3}$.

A lesão nervosa periférica é a principal complicação nesse tipo de fratura, podendo variar amplamente em extensão e severidade. No caso da lesão do nervo radial, pode ocorrer a incapacidade de extensão do cotovelo, punho e da função preensora da mão, provocando significativas perdas funcionais ${ }^{4}$, as quais podem ser recuperadas se os axônios lesados crescerem, alcançarem 0 coto distal e restabelecerem conexões funcionais com 0 alvo muscular apropriado 5 .

Como uma das principais alterações que ocorrem no músculo após a lesão nervosa, cita-se a atrofia muscular que, uma vez instalada, atua como barreira ao crescimento axonal durante a reinervação muscular. Sendo assim, o uso da eletroestimulação e de cinesioterapia de fortalecimento muscular é rotineiro no tratamento fisioterapêutico, tendo como objetivo minimizar ou impedir a atrofia muscular e, assim, favorecer a recuperação da lesão nervosa periférica ${ }^{6}$.

Dentre os recursos da eletroterapia eficazes nesse tipo de lesão, o mais utilizado é a Estimulação Elétrica Funcional (FES), pois esse induz potenciais de ação no nervo motor, provocando ativação de unidades motoras. Além disso, produz efeitos como fortalecimento da musculatura estimulada e facilitação do controle motor voluntário ${ }^{7}$.

Dessa forma, a realização desse estudo justifica-se pelos resultados positivos alcançados por essas técnicas, no tratamento de lesão nervosa associada à fratura cominutiva e atrofia e/ou hipotonia muscular, neste estudo de caso, sendo sugestiva a sua aplicação em estudos com maior número de amostragem.

\section{Metodologia}

Realizou-se um estudo de caso, com análise descritiva, por meio de dados de prontuário, da avaliação fisioterapêutica e da descrição das técnicas utilizadas durante 0 tratamento, o qual foi efetivado no Ambulatório de Fisioterapia do Hospital Universitário de Santa Maria (HUSM) - RS, no período de outubro de 2012 a janeiro de 2013, através da disciplina Fisioterapia em Ortopedia e Traumatologia, ofertada no sexto semestre do curso de Fisioterapia, da Universidade Federal de Santa Maria.

Desde o início da terapia, a paciente foi esclarecida a respeito dos objetivos e procedimentos que seriam realizados durante o tratamento. Dessa forma, a paciente assinou

Saúde (Santa Maria), v.39, n.2, p.71-78, 2013. Reabilitaçăo de lesão do nervo radial após fratura cominutiva de úmero: um estudo de caso um termo de consentimento livre e esclarecido autorizando o uso de seu caso clínico e condições pós-tratamento para publicação científica, estando livre para abandono de tratamento sem qualquer penalidade, bem como ter sua identidade preservada. 
A avaliação fisioterapêutica foi composta por uma ficha de anamnese, análise de prontuário e exame físico, onde foram realizados testes de força muscular utilizando como referência a graduação de força de 0 a 5 . A amplitude de movimento foi avaliada pré e pósintervenção através da goniometria. Foi realizado ainda o teste de sensibilidade (superficial e profunda) e palpação do trajeto do nervo radial.

\section{$\underline{\text { Relato de Caso }}$}

Paciente do sexo feminino M.C.V, 41 anos, professora, diagnóstico clínico baseado em lesão do nervo radial, por meio de uma fratura cominutiva do úmero, em ambos os braços, gerada por acidente automobilístico, além de sofrer amputação transfemural esquerda. Relata ter permanecido 39 dias em hospitalização, onde realizou tratamento cirúrgico com pinos e placas no braço direito e haste intra-medular no braço esquerdo. Iniciou o tratamento com fisioterapia 04 meses após 0 acidente, em Porto Alegre, com frequência semanal, porém somente 07 meses após o acidente iniciou seu tratamento fisioterapêutico no Ambulatório de Fisioterapia do Hospital Universitário de Santa Maria.

Foram solicitados exames complementares para maior controle da consolidação das fraturas, que evidenciaram redução por osteossintese, assim como calosidades em desenvolvimento nos úmeros.

A paciente apresentava-se em bom estado geral, fazendo uso de cadeira de rodas, punho em flexão ou "mão caída", uso de talas, leve assimetria nas clavículas devido a uma possível anquilose (demonstrada no exame radiográfico), ausência de sensação dolorosa e presença leve de sensação tátil à palpação, perda quase total do movimento de extensão de punho.

Através da goniometria realizada na avaliação inicial, os seguintes ângulos foram aferidos:

- no membro superior direito: $90^{\circ}$ de flexão do ombro, $20^{\circ}$ de hiperextensão do ombro, $140^{\circ}$ de abdução do ombro, $37^{\circ}$ de adução do ombro; $125^{\circ}$ de flexão de cotovelo; enquanto 0 punho apresentou maior limitação, sem movimento de extensão, em flexão de $65^{\circ}, 10^{\circ}$ de desvio ulnar, $5^{\circ}$ de desvio radial, articulação metacarpofalângica (MCF) em flexão de $50^{\circ}$.

- no membro superior esquerdo: $90^{\circ}$ de flexão de ombro, $22^{\circ}$ de hiperextensão de ombro, $78^{\circ}$ de abdução de ombro, $36^{\circ}$ de adução de ombro; $140^{\circ}$ de flexão de cotovelo; desvio ulnar de $15^{\circ}$ e desvio radial de $7^{\circ}$ enquanto o punho e articulação MCF permaneceram com as mesmas angulações do membro superior direito.

No teste de força muscular, os extensores de punho apresentaram força grau 01 , enquanto os flexores de punho, rotadores internos e externos de ombro, flexores de ombro, abdutores e adutores de ombro força grau 04.

Sendo assim, o diagnóstico fisioterapêutico compreendeu hipotrofia muscular, diminuição da amplitude de movimento e de força muscular, principalmente de extensores do punho.

A terapia foi composta por 2 sessões semanais, com duração de 1 hora cada sessão, totalizando 16 sessões, sendo o tratamento fisioterapêutico progressivo. As primeiras sessões eram iniciadas com aplicação de 15 minutos de Estimulação Elétrica Funcional (FES) nos músculos extensores do antebraço, com os parâmetros: frequência $50 \mathrm{~Hz}$, tempo de subida de 6 segundos, tempo de descida de 6 segundos, tempo ON de 10 segundos, tempo OFF de

Rev. Saúde (Santa Maria), Santa Maria, v.39, n.2, p.71-78, Jul./Dez.2013 Freire, A. B.; et al. 
20 segundos, intensidade que variou de 47 , no início da terapia, até 90 , já na metade do tratamento. Essa modalidade era utilizada com o objetivo de estimular a inervação radial, 0 fortalecimento muscular, manutenção da ADM, retardo de atrofias musculares e manutenção da tonicidade da musculatura. Entre a $3^{\mathrm{a}}$ e $11^{\mathrm{a}}$ sessões, para a finalização das mesmas, foram aplicadas bandagens funcionais com o intuito de estimular a extensão e desvio ulnar do punho, através do estimulo de receptores sensitivos e motores, além dos proprioceptivos, e posicionamento do punho nas posições anteriormente citados.

Foi realizada cinesioterapia e exercícios de fortalecimento muscular progressivo para os membros superiores com 0 uso de pesos, halteres, bastão e theratub. Inicialmente as sessões eram compostas de 3 séries de 8 repetições para cada grupo muscular, sendo $1,5 \mathrm{~kg}$ para flexores de ombro; $0,5 \mathrm{~kg}$ para abdutores de ombro (a $45^{\circ}$ de abdução); $2 \mathrm{~kg}$ para flexores de cotovelo; theratub para extensores de cotovelo; $0,5 \mathrm{~kg}$ para prono/supino; isométricos leves para extensores de punho.

Com a melhora da capacidade da musculatura, os exercícios evoluíram da seguinte forma: 3 séries de 10 repetições, 2 kg na flexão de ombro; 2 kg na abdução de ombro; $2,5 \mathrm{~kg}$ na flexão de cotovelo; extensão de cotovelo com theratub verde; $1 \mathrm{~kg}$ para prono/supino; $1 \mathrm{~kg}$ para desvio radial e ulnar; $1 \mathrm{~kg}$ para flexo/extensão de punho com auxílio proprioceptivo no antebraço; isométricos de extensão de punho com $1 \mathrm{~kg}$ e sustentação de 5 segundos. Ao final do tratamento os exercícios constavam de: 3 séries de 6 repetições, $3 \mathrm{~kg}$ para flexão de ombro; $3 \mathrm{~kg}$ para abdução de ombro; $3 \mathrm{~kg}$ para flexão de cotovelo; $3 \mathrm{~kg}$ para extensão de cotovelo; 3 séries de 10 repetições para os seguintes exercícios: $1 \mathrm{~kg}$ para prono/supino; $1 \mathrm{~kg}$ para desvio radial e ulnar; $1 \mathrm{~kg}$ para flexo/extensão de punho; isométricos para extensão de punho com sustentação de 10 segundos.

Todos os exercícios eram executados de forma intercalada para um melhor rendimento do tratamento, objetivando estimular a inervação radial, estimular e fortalecer a musculatura dos membros superiores, restaurar a ADM das grandes e pequenas articulações, bem como retornar ao nível funcional da paciente, melhorando independência social e qualidade de vida.

\section{Resultados e Discussão}

A avaliação final evidenciou que a paciente apresentou boa evolução, demonstrando ganho de força muscular durante as atividades e mantendo o desempenho com a duplicação da carga e, posteriormente, redução das repetições com carga ainda maior, visando 0 fortalecimento muscular.

Durante a regeneração do nervo radial, o uso da corrente FES mostrou-se eficaz na estimulação da musculatura extensora de punho, de modo que na metade do tratamento foi atingida máxima intensidade no aparelho, não sendo mais necessária a continuidade dessa modalidade.

Para o tratamento da paciente, as sessões combinavam o uso de eletroestimulação, cinesioterapia e exercícios de fortalecimento, visando desde o ganho de amplitude de movimento, até o fortalecimento dessa musculatura, para aliviar as consequências advindas da lesão. Para Petronic et al. (2003), ${ }^{8}$ as lesões das extremidades podem ser acompanhadas por várias complicações neuromusculares, como a citada lesão dos nervos periféricos. Em seu estudo realizado com 16 pacientes com lesão nervosa periférica, três deles com nervo cominutiva de úmero: um estudo 
radial comprometido, foram utilizados métodos de tratamento condizentes com o presente estudo, ou seja, eletroterapia, associada à cinesioterapia e com o avançar do tratamento uso de órteses estáticas e dinâmicas. Os autores concluíram que após a recuperação é possível não haver sequelas se houver uma adequada união entre os seguintes fatores: tratamento cirúrgico precoce e adequado, realização e manutenção da fisioterapia e um trabalho integrado, em equipe, entre o neurocirurgião, neurologista e o fisioterapeuta.

Com 0 avanço das sessões, foi observado ganho de força muscular dos membros superiores da paciente. Concordando assim com os achados de Fagundes et al. (2005), ${ }^{9} 0$ qual relata que a estimulação elétrica muscular tem sido empregada após lesões nervosas periféricas com o objetivo de minimizar a atrofia e a fraqueza muscular, pois as fibras musculares possuem plasticidade, sendo o músculo capaz de sofrer adaptações à desenervação e à estimulação elétrica. Esse trabalho concluiu que a estimulação elétrica de baixa frequência é capaz de minimizar a atrofia das fibras musculares e a proliferação de tecido conjuntivo no músculo desenervado, mantendo sua força e resistência.

De acordo com Dow et al., ${ }^{10,11} \mathrm{em}$ trabalhos de 2004 e 2005, descreve que o peso da massa de músculos desenervados, mas que são submetidos a eletroestimulação, apresentaram valores significativamente maiores ao de músculos desenervados sem intervenção. Nesses estudos, os autores destacam que há uma relação direta entre o número de contrações geradas por dia e a distribuição delas com o efeito trófico da eletroestimulação em músculos sem inervação, sendo indicadas de 200 a 800 contrações/dia, com distribuição contínua e constante ao longo do dia. Além disso, Tomori et al. (2010) ${ }^{12}$ e Kanaya et al. $(1992)^{13}$ também verificaram o efeito da eletroestimulação para o trofismo muscular na fase de desenervação, com um protocolo de intervenção não invasivo e com duração fásica menor do que oito horas por dia. Os resultados de ambos estudos apontaram o efeito benéfico da eletroestimulação para minimizar a atrofia muscular, fato que também foi observado no presente estudo. Tomori et al. $(2010)^{12}$ relata que a intensidade da corrente tem relação direta com os efeitos da eletroestimulação, não recomendando intensidades muito baixas ou muito altas.

Segundo Cavalcante et al. (2012), ${ }^{6}$ um protocolo de eletroestimulação adequado à característica de baixa excitabilidade do músculo desenervado, ou seja, baixa amplitude ou duração de pulso maior, ou com períodos suficientes para produzir no mínimo 50 contrações por dia, mostram-se eficazes em minimizar a perda do trofismo em músculos que perderam sua inervação, podendo-se afirmar que a eletroestimulação apresenta efeito trófico.

Além disso, no presente estudo houve ganho na amplitude de movimento, como é demonstrado na Tabela 1.

Rev. Saúde (Santa Maria), Santa Maria, v.39, n.2, 
Tabela 1 - Comparação da goniometria do MSD e MSE entre a avaliação inicial e avaliação final

\begin{tabular}{ccccccc}
\hline $\begin{array}{c}\text { Ângulos/ Movimentos } \\
\text { Avaliados }\end{array}$ & $\begin{array}{c}\text { MSD } \\
\text { avaliação } \\
\text { inicial }\end{array}$ & $\begin{array}{c}\text { MSD } \\
\text { avaliação } \\
\text { final }\end{array}$ & $\begin{array}{c}\text { Ganhos } \\
\text { obtidos }\end{array}$ & $\begin{array}{c}\text { MSE } \\
\text { avaliação } \\
\text { inicial }\end{array}$ & $\begin{array}{c}\text { MSE } \\
\text { avaliação } \\
\text { final }\end{array}$ & $\begin{array}{c}\text { Ganhos } \\
\text { obtidos }\end{array}$ \\
\hline Flexão do ombro & $90^{\circ}$ & $145^{\circ}$ & $55^{\circ}$ & $90^{\circ}$ & $136^{\circ}$ & $46^{\circ}$ \\
Hiperextensão do ombro & $20^{\circ}$ & $35^{\circ}$ & $15^{\circ}$ & $22^{\circ}$ & $30^{\circ}$ & $8^{\circ}$ \\
Abdução do ombro & $140^{\circ}$ & $155^{\circ}$ & $15^{\circ}$ & $78^{\circ}$ & $143^{\circ}$ & $65^{\circ}$ \\
Adução do ombro & $37^{\circ}$ & $45^{\circ}$ & $8^{\circ}$ & $36^{\circ}$ & $45^{\circ}$ & $9^{\circ}$ \\
Flexão de cotovelo & $125^{\circ}$ & $145^{\circ}$ & $20^{\circ}$ & $135^{\circ}$ & $145^{\circ}$ & $10^{\circ}$ \\
Desvio ulnar & $10^{\circ}$ & $25^{\circ}$ & $15^{\circ}$ & $5^{\circ}$ & $20^{\circ}$ & $15^{\circ}$ \\
Desvio radial & $5^{\circ}$ & $12^{\circ}$ & $7^{\circ}$ & $5^{\circ}$ & $10^{\circ}$ & $5^{\circ}$ \\
Hiperextensão de punho & $65^{\circ}$ & $20^{\circ}$ & $45^{\circ}$ & $65^{\circ}$ & $13^{\circ}$ & $52^{\circ}$ \\
Articulações MCF & $90^{\circ}$ & $0^{\circ}$ & $90^{\circ}$ & $50^{\circ}$ & $2^{\circ}$ & $48^{\circ}$ \\
\hline
\end{tabular}

Os ganhos nas ADM's demonstrados na tabela 1 concordam com os resultados do estudo de Corrêa et al. (2009), ${ }^{14}$ no qual participaram três pacientes do sexo masculino portadores de hemiplegia após AVE e com subluxação na articulação do ombro, os quais foram submetidos ao tratamento com fisioterapia convencional e aplicação da eletroestimulação funcional no membro superior hemiplégico duas vezes por semana, com duração de 60 a 70 minutos por sessão, durante cinco semanas (dez sessões). Os valores das medidas após o tratamento apontaram vantagens no uso da fisioterapia associada à eletroestimulação funcional, ao comparar com as medidas iniciais de amplitude de movimento.

Os efeitos positivos da eletroestimulação na força muscular (principalmente na força isométrica de extensão do punho), tônus (redução do tônus dos músculos flexores do punho), função e uso do membro na rotina diária foram demonstrados com forte evidência na revisão de literatura de Arantes et al. (2007) ${ }^{7}$. Outros trabalhos realizados por esses autores demonstraram efeitos positivos no uso da eletroestimulação para punho e dedos, o que sugere que essa terapia seja eficaz para a promoção de função do membro superior afetado, concordando com os achados do presente estudo.

Segundo resultados do estudo de Kkhir e Alekhin $(2011)^{15}$ que utilizaram 52 pacientes com lesões do nervo radial, mesmo com os tratamentos, a reinervação e a recuperação funcional dos músculos desenervados, mas sem secção do nervo, é lenta.

Saúde (Santa Maria), v.39, n.2, p.71-78, 2013. Reabilitaçăo de lesão do nervo radial após fratura cominutiva de úmero: um estudo de caso ISSN 2236-58359

\section{Conclusão}

Com este relato de caso, pode-se observar o resultado positivo da utilização de métodos fisioterapêuticos em pacientes que sofreram lesão de nervo radial, a fim de restaurar amplitude de movimento e força muscular. A utilização do FES como estímulo para a estimulação muscular durante a regeneração da inervação radial, associada ao estímulo proprioceptivo e cinesioterapia progressiva, com o uso de halteres, pesos, bastão e theratub 
mostraram ser eficazes na recuperação da lesão nervosa e da musculatura da paciente. Sendo assim, o tratamento proporcionou 0 retorno as suas atividades de vida diária, possibilitando maior independência funcional e melhor qualidade de vida.

Portanto, esse estudo demonstra que a utilização dessas técnicas fisioterapêuticas é eficaz na reabilitação de pacientes com lesão nervosa, possibilitando sua aplicação em estudos com maior amostra.

\section{Referências bibliográficas}

1. Calil, A. M.; Sallum, E. A.; Domingues, C. A.; Nogueira, L. S. Mapeamento das lesões em vítimas de acidentes de trânsito: Revisão Sistemática da literatura. Rev Latino-am Enfermagem 2009; 17(1): 121-127.

2. Itami, L. T.; Faro, A. C. M.; Meneghin, P.; Leite, R. C. B. O.; Silveira, C. T. Adultos com fraturas: das implicações funcionais e cirúrgicas à educação em saúde. Rev Esc Enferm USP 2009; 43(Esp 2):1238-43.

3. Azevedo, M. C.; Azevedo, G. M.; Hayashi, A. Y.; Nascimento, P. E. D. Tratamento de fraturas e complicações pós-traumáticas do úmero com fixadores externos osteoline®: uma opção de tratamento. Rev Bras Ortop. 2011;46(4):390-97

4. Batista, K. T.; Araújo, H. J.; Araújo, E. A. Resultados do reparo cirúrgico tardio das lesões traumáticas do nervo radial. Rev. Bras. Cir. Plást. 2010; 25(3): 458-64.

5. Possamai, F.; Pacheco, D. R.; Santos, T. S.; André, E. S. Repercussões morfológicas e funcionais do exercício sobre a regeneração nervosa periférica. Fisioter. Mov. 2012; 25(3):617-627.

6. Cavalcante, E. V. V.; Silva, L. G. M.; Montenegro, E. J. N.; Filho, N. T. P. Efeito da eletroestimulação no músculo desenervado de animais: revisão sistemática. Fisioter. Mov. 2012; 25(3):669-678.

7. Arantes, N. F.; Vaz, D. V.; Mancini, M. C.; Pereira, M. S. D. C.; Pinto, F. P.; Pinto, T. P. S. Efeitos da estimulação elétrica funcional nos músculos do punho e dedos em indivíduos hemiparéticos: uma revisão sistemática da literatura. Ver. Brás. Fisioter. 2007;11(6):89-93.

8. Petronic, l.; Marsavelski, A.; Nikolic, G.; Cirovic, D. Postoperative rehabilitation in patients with peripheral nerve lesions. Acta Chir lugosl. 2003;50(1):83-86.

9. Fagundes, K. C. B. G.; Polacow, M. L. O.; Guirro, R. R. J.; Campos, G. E. R.; Somazz, M. C.; Pinto, V. F.; Fuentes, C. B.; Teodori, R. M. Analise morfométrica dos tecidos muscular e conjuntivo após desenervação e estimulação elétrica de baixa frequência. Rev. Brás. Fisioter. 2005;9(2):235-241.

10. Dow, D. E.; Cederna, P. S.; Hassett, C. A.; Kostrominova, T. Y.; Faulkner, J. A.; Dennis, R. G. Number of contractions to maintain mass and force of a denervated rat muscles. Muscle Nerve. 2004:30(1):77-86.

11. Dow, D. E.; Faulkner, J. A.; Dennis, R. G. Distribution of rest periods between electrically generated contractions in denervated muscles of rats. Artif Organs. 2005;29(6):432-5.

12. Tomori, K.; Ohta, Y.; Nishizawa, T.; Tamaki, H.; Takekura, H. Low-intensity electrical stimulation ameliorates disruption of transverse tubules and neuromuscular junctional architecture in denervated rat skeletal muscle fibers. J Muscle Res Cell Motil. 2010;31(3):195-205.

13. Kanaya, F.; Tajima T. Effect of electrostimulation on denervated muscle. Clin Orthop Relat Res. 1992;283:296-301.

14. Corrêa, J. B.; Borges, H. C.; Lucareli, P. R. G.; Liebano, R. E. Estimulação elétrica funcional na subluxação crônica do ombro após acidente vascular encefálico: relato de casos. Fisioter. Pesqui. 2009;16(1):89-93.

Rev. Saúde (Santa Maria), Santa Maria, v.39, n.2, p.71-78, Jul./Dez.2013. Freire, A. B.; et al. 
15. Kkhir, B. M.; Alekhin, A.l.; Golubev, V.G.; lulov, V.V. The optimal treatment algorithm of radial nerve injuries. Khirurgiia (Mosk). 2011;(12):23-28.

\section{Ariane Bôlla Freire}

Endereço para correspondência - Rua João Batista da Cruz Jobim, 235. Bairro Medianeira, Santa Maria, CEP:97060330, RS, Brasil.

E-mail: aribfreire@hotmail.com

Currículo Lattes: http://lattes.cnpq.br/5200221092017780

Recebido em 03 de março de 2013.

Aprovado em de 09 setembro de 2013. 